\title{
Modèles continus pour les structures rocheuses discontinues
}

\section{J. SULEM}

Centre d'enseignement et de recherche en mécanique des sols École nationale des ponts et chaussées/Laboratoire central des ponts et chaussées

6-8, avenue Blaise-Pascal Cité Descartes Champs-sur-Marne 77455 Marne-la-Vallée Cedex 2, France sulem@cermes.enpc.fr

\section{CERROLAZA}

Instituto de materiales y modelos estructurales Universitad central de Venezuela, IMME

Facultad de Ingenieria

Universitad central de Venezuela Apartado Postal 50361 Caracas 1050, Venezuela mcerrola@reacciun.ve
On présente un modèle continu de Cosserat pour les structures de blocs rocheux. L'introduction d'une rotation de Cosserat et de couples de contraintes permet de prendre en compte la rotation individuelle des blocs et leur rigidité en flexion. On montre que le domaine de valídité de cette homogénéisation est étendu au cas de structures dont les dimensions sont un faible multiple de la taille des blocs. Par ailleurs différents modes de plastification par glissement inter-blocs ou pivotement des blocs sont introduits dans un modèle de plasticité multicritères. Ce modèle est illustré par l'étude de la réponse dynamique d'une structure de maçonnerie et par deux exemples de géotechnique, la stabilité d'une fondation souple et la stabilité d'une pente dans un massif constitué de blocs rocheux.

Mots-clés : roches fracturées, stabilité des pentes, homogénéisation, milieux de Cosserat.

The discontinuous structure of blocky rock is approximated by an equivalent Cosserat continuum. This implies the introduction of couple stresses and internal rotations which model the relative rotations between blocks and the bending stiffness of the blociss. It is shown that the domain of validity of the homogenisation is extended to the case of structures with few blocks. The advantage of the Cosserat homogenisation for blocky structure is also that various failure modes such as interblock slip and block tilting can be easily described through a multi-criteria plasticity model. This model is illustrated by the analysis of the dynamic response of a masonry structure and by two examples of soft foundation and slope stability in blocky rock.

Key words : jointed rock, slope stability, homogenisation. 


\section{Introduction}

Il est courant d'observer que sous l'effet des contraintes tectoniques, un massif rocheux présente un réseau régulier de fractures lui donnant du point de vue macroscopique l'apparence d'une structure de blocs rocheux. La mécanique des milieux continus classiques offre un cadre approprié et bien établi lorsqu'il s'agit de modéliser un processus de déformation dont la longueur d'onde dominante est grande comparée à la taille caractéristique des discontinuités de la structure considérée. Les méthodes d'homogénéisation permettent de définir les caractéristiques mécaniques d'un milieu continu équivalent, à grande échelle, au matériau discontinu initial (Bakhalov et Panasenko, 1989). L'intérêt de développer une approche continue pour un milieu hétérogène ou discontinu réside dans le fait que les approches « discrètes )) (Cundall et Hart, 1992) conduisent à des calculs numériques considérables lorsque le nombre des discontinuités ou des hétérogénéités augmente. Parmi les avantages d'une représentation par milieu continu équivalent, on peut également citer le fait que le maillage par éléments finis utilisé pour la résolution d'un problème aux limites est indépendant de la géométrie des discontinuités. Enfin, dans certains cas, comme pour des milieux rocheux largement fracturés, on ne dispose pas toujours d'information précise sur les réseaux de failles et de joints et seules des valeurs moyennes des modules de déformation peuvent être utilisées.

Le milieu continu homogénéisé ne peut reproduire que le comportement à grande échelle du matériau réel et c'est là sa principale limitation. On peut cependant étendre le domaine de validité de l'approche continue en ayant recours à des modèles de milieux continus d'ordres supérieurs ou milieux continus généralisés qui possèdent dans leur formulation des degrés de liberté cinématiques supplémentaires comme les milieux de Cosserat (Cosserat, 1909) et/ou des gradients de déformation d'ordres supérieurs. Les modèles de milieux continus généralisés contiennent dans leur description une ou plusieurs longueurs internes reliées à la microstructure du matériau. Dans cet article, on présente tout d'abord le cadre général de la mécanique des milieux continus généralisés à travers le formalisme de Mindlin (1964). Les milieux stratifiés et les structures de blocs représentent deux exemples pour lesquels la théorie de Cosserat est tout à fait adaptée. Ces deux exemples sont présentés et illustrés pour le cas de structures de maçonnerie et pour celui de la stabilité de pentes rocheuses.

\section{2}

\section{Milieux continus généralisés et formalisme de Mindlin}

La description de la statique et de la cinématique des milieux continus possédant une microstructure lou milieux continus généralisés) a été présentée d'une manière systématique par Germain (1973a, b) à partir de l'application du principe des puissances virtuelles en suivant le formalisme proposé par Mindlin (1964).

Dans une description classique, un milieu continu est une distribution de points matériels caractérisés par une position X et une vitesse v. Dans une théorie qui prend en compte la microstructure du matériau, on associe à chaque particule matérielle un continuum $\mathrm{C}(\mathrm{X})$ déformable autour du point $\mathrm{X}$ appelé microvolume. Les tenseurs de vitesse de macrodéformation $\varepsilon$ et de macrorotation $\omega$ sont définis comme la partie symétrique et la partie antisymétrique du gradient du champ de vitesse:

$$
\varepsilon_{i j}=\frac{1}{2}\left(\partial_{j} v_{j}+\partial_{j} v_{j}\right), \omega_{i j}=\frac{1}{2}\left(\partial_{j} v_{j}-\partial_{j} v_{j}\right)
$$

On définit un tenseur de vitesse de microdéformation $\psi$ correspondant à la déformation du microvolume $\mathrm{C}(\mathrm{X})$ supposée homogène. La vitesse déformation relative $\gamma$ est alors définie comme la différence entre le gradient de macrovitesse et la vitesse de microdéformation

$$
\gamma=\nabla v-\Psi
$$

Enfin, on définit le tenseur d'ordre 3 de gradient de vitesse de microdéformation

$$
\kappa=\nabla \Psi
$$

Les quantitês statistiques duales dérivent alors de l'application du principe des puissances virtuelles et de l'expression de la puissance des efforts intérieurs:

$$
\delta w^{(i)}=\tau \delta \varepsilon_{i j}+\alpha_{j i} \delta \gamma_{j}+\mu_{i j k} \delta \kappa_{i k}
$$

où $\tau$ est le tenseur de contrainte de Cauchy (symétrique) associé au tenseur de vitesse de macrodéformation $\varepsilon ; \alpha$ est le tenseur de contraintes relatives associé au tenseur de vitesse de déformation relative $\gamma ; \mu$ est le tenseur de double contraintes associé au gradient de vitesse de microdéformation $\kappa$ (tenseur d'ordre 3 ).

On peut écrire la puissance des efforts intérieurs (4) sous la forme

$$
\delta w^{i i}=\sigma_{j} \partial_{j} v_{i}-\alpha_{j} \delta \psi_{i j}+\mu_{i j k} \delta \kappa_{i j k}
$$

où $\sigma=\tau+\alpha$ est le tenseur de contraintes totales,

Le principe des puissances virtuelles permet d'écrire les équations d'équilibre pour un milieu continu généralisé sous la forme

$$
\begin{gathered}
\partial \sigma_{j i}+f=0 \\
\alpha_{j k}+\partial \mu_{j i k}+\Phi_{j k}=0
\end{gathered}
$$

où fet $\Phi$ représente respectivement les forces de volume et les doubles forces de volume. Du principe des puissances virtuelles on déduit également les conditions aux frontières. On suppose que la frontière $\partial \mathrm{V}$ du volume $\mathrm{V}$ considéré est divisée en deux parties complémentaires. $\left\{\partial V_{u} \partial V_{\sigma}\right\}$ et $\left\{\partial V_{v} \partial V_{u}\right\}$ respectivement, telles que :

$$
\begin{aligned}
& \text { sur } \partial V_{\nu}: u=\bar{Q} \\
& \text { sur } \partial V_{v}: \psi=\bar{\psi} \\
& \text { sur } \partial V_{\sigma}: \sigma \cdot n=\bar{t} \\
& \text { sur } \partial V_{\mu}: \mu \cdot n=\bar{T}
\end{aligned}
$$

Le formalisme ci-dessus permet d'obtenir les équations de base d'un milieu de Cosserat. Un milieu de Cosserat est un milieu micropolaire pour lequel le microvolume $C(X)$ est un corps rigide. Par conséquent un milieu de Cosserat est un milieu continu qui associe à chaque point matériel une vitesse de translation et une vitesse de rotation propre $\omega^{c}$ différente de la rotation d'ensemble $\omega$. Dans la suite l'indice c sera utilisé pour distinguer la rotation de Cosserat de la rotation macroscopique. Par conséquent, le tenseur de microdéformation est purement antisymétrique et on l'identifie à $\omega^{\mathrm{c}}$, et le gradient de microrotation, également antisymétrique, représente un tenseur de courbure:

$$
\begin{gathered}
\psi_{i j}=\psi_{i j i}=\omega_{i j}^{c} \\
\gamma_{i j}=\varepsilon_{i j}+\left(\omega_{i j}-\omega_{i j}^{c}\right) \\
\kappa_{i j k}=\partial_{i j} \omega_{j k}^{c}
\end{gathered}
$$


La partie symétrique du tenseur de déformation relative $\gamma$ coïncide avec le tenseur de macrodéformation $\varepsilon$ et la différence entre macro- et microdéformation est purement antisymétrique et représente la différence entre la rotation individuelle du microvolume et la rotation d'ensemble du domaine autour du microvolume.

Les quantités statiques qui apparaissent dans un milieu de Cosserat se déduisent de l'application du principe des puissances virtuelles. Le tenseur de contraintes relatives $\alpha$ coincide avec la partie antisymétrique du tenseur de contraintes totales $\sigma$. On simplifie les notations en introduisant les adjoints des différents tenseurs.

$\omega_{j}^{c}=-e_{j k} \omega_{k}{ }^{c}, \mu_{j k}=-e_{j,} m_{j u}, \Phi_{j}=-e_{i j k} \Phi_{k}, T_{j}=-e_{i j k} M_{k}$

où e est le tenseur de permutation défini par

$e_{j k}= \begin{cases}1 & \text { si ijk est une permutation paire de } 123 \\ -1 & \text { si ijk est une permutation impaire de } 123 \\ 0 & \text { sinon }\end{cases}$

$\omega^{c}$ est le vecteur de rotation de Cosserat. $\Phi$ et $M$ sont des moments et $m$ est la densité de couple de contraintes.

\section{3}

\section{Un exemple d'application des milieux de Cosserat : les massifs rocheux stratifiés}

Les massifs rocheux sont le plus souvent fracturés. Bien que le réseau de fractures puisse être complexe, on peut généralement observer une certaine régularité des discontinuités, i.e. des directions préférentielles d'orientation de la fracturation. Les fractures sont souvent rugueuses et remplies par un matériau plus tendre, ce qui crée une résistance au glissement relatif le long des deux faces de la discontinuité. Des approches similaires à celles développées pour les matériaux composites ont été appliquées pour représenter les roches stratifiées par un milieu continu équivalent anisotrope (Salamon, 1968). La taille du volume élémentaire du milieu homogénéisé est grande comparée à l'épaisseur des couches individuelles. Cette approche est valide tant qu'il n'y a pas de glissement interne le long des couches.

Considérons un milieu plan infini constitué de couches élastiques planes parallèles identiques d'épaisseur $2 h$. Soit $u$ et $w$ les composantes du déplacement. On suppose que le poids des couches supérieures empêche l'ouverture des joints. Pour une loi d'interface élastique le glissement tangentiel le long des joints s'écrit de façon linéaire :

$$
\tau=\frac{k_{s}}{2 h}\left(u_{n}^{-}-u_{n+1}^{*}\right)
$$

La structure stratifiée du matériau conduit à une double échelle : une échelle macroscopique où l'élément de volume représentatif peut être représenté par le rectangle $A B C D$ (ou macro-élément) dont les côtés sont grands par rapport à l'épaisseur des couches et une échelle microscopique représentée par le rectangle abcd (ou micro-élément) dont les côtés sont petits comparés à l'épaisseur des couches (Fig. 1).

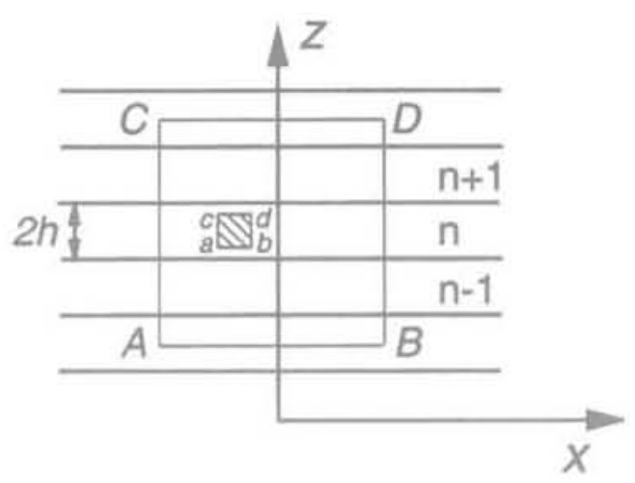

FG.1 Schéma d'un milieu stratifié.

Si la symétrie des contraintes tangentielles est respectée pour le micro-élément, celle-ci est violée pour le macro-élément dès qu'il y a glissement le long des frontières des couches. L'équilibre est alors assuré par l'apparition de moments de flexion (Fig, 2) :

$$
\frac{d M}{d x}+\tau_{z x}-\tau_{2 x}=0
$$

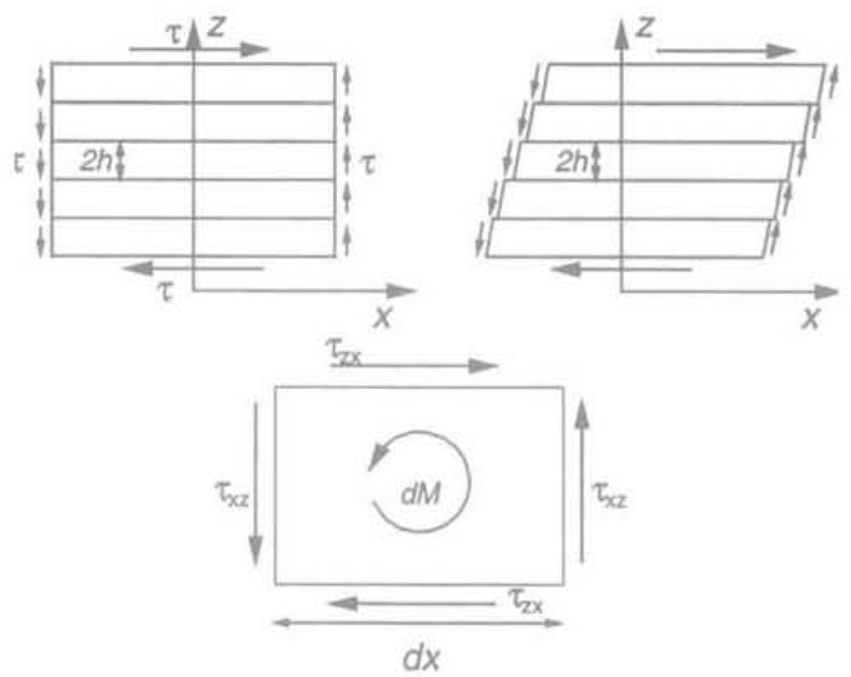

FG. 2 Rupture de symétrie des contraintes tangentielles.

Ces moments de flexion des couches conduisent à une rotation locale comme degré de liberté supplémentaire de la cinématique du macro-élément. Cette rotation locale ne peut être prise en compte dans le cadre de la mécanique des milieux continus classiques et nécessite d'avoir recours à la théorie de Cosserat. Par ailleurs, ces moments de flexion donnent naissance à de forts gradients dans la distribution locale des contraintes. Cela affecte sensiblement à la fois le mode de déformation et le mode de rupture des différentes couches. 
Dans un milieu de Cosserat bidimensionnel, la cinématique de chaque point matériel est définie par deux degrés de liberté de translation $(u, w)$ et un degré de liberté de rotation $\omega^{\mathrm{c}}$ (Fig. 3). Le tenseur de déformation relative et le vecteur courbure (équation 8) s'expriment de la façon suivante :

$$
\begin{aligned}
& \gamma_{x x}=\frac{\partial u}{\partial x} ; \gamma_{x z}=\frac{\partial u}{\partial z}+\omega^{c} \\
& \gamma_{z z}=\frac{\partial w}{\partial z} ; \gamma_{z x}=\frac{\partial w}{\partial x}-\omega^{c} \\
& \kappa_{x}=\frac{\partial \omega^{c}}{\partial x} ; \kappa_{z}=\frac{\partial \omega^{c}}{\partial z}
\end{aligned}
$$
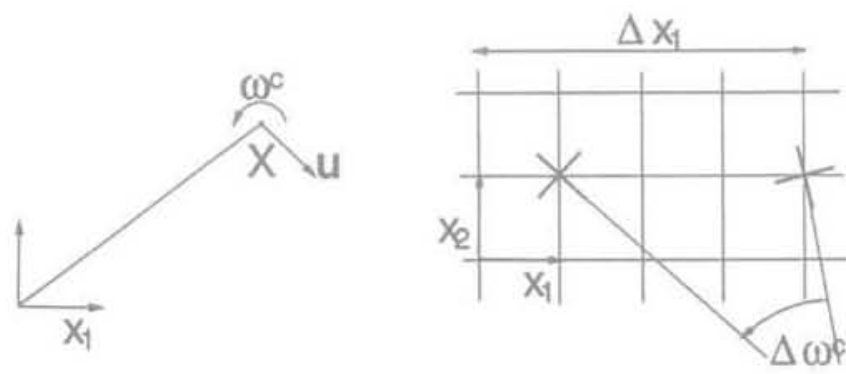

คG.3 Quantités cinématiques dans un milieu de Cosserat.

Les équations d'équilibre (6) en l'absence de forces et de couples de volume deviennent:

$$
\begin{aligned}
& \frac{\partial \sigma_{x x}}{\partial x}+\frac{\partial \sigma_{x z}}{\partial z}=0 \\
& \frac{\partial \sigma_{z x}}{\partial x}+\frac{\partial \sigma_{z z}}{\partial z}=0 \\
& \frac{\partial m_{x}}{\partial x}+\frac{\partial m_{z}}{\partial z}+\sigma_{z x}-\sigma_{z x}=0
\end{aligned}
$$

Les deux premières equations d'équilibre sont formellement les mêmes que pour un milieu continu classique. Une équation d'équilibre supplémentaire pour les couples de contraintes est introduite (Fig. 4).

Les équations de comportement d'un milieu de Cosserat bidimensionnel élastique isotrope s'écrivent (Schaefer, 1962; Vardoulakis et Sulem, 1995) :

$$
\begin{gathered}
\sigma_{11}=(K+G) \gamma_{11}+(K-G) \gamma_{22} \\
\sigma_{22}=(K-G) \gamma_{11}+(K+G) \gamma_{22} \\
\sigma_{12}=[G+G] \gamma_{12}+(G-G) \gamma_{21} \\
\sigma_{21}=\left(G-G_{c}\right) \gamma_{12}+(G+G) \gamma_{21} \\
m_{1}=M \kappa_{1} \\
m_{2}=M \kappa_{2}
\end{gathered}
$$

Dans les relations de comportement (15), $K$ est le module de compressibilité bidimensionnel et $G$ est le module de cisaillement qui relie la partie symétrique de la déformation déviatorique à la partie symétrique de la contrainte déviato-
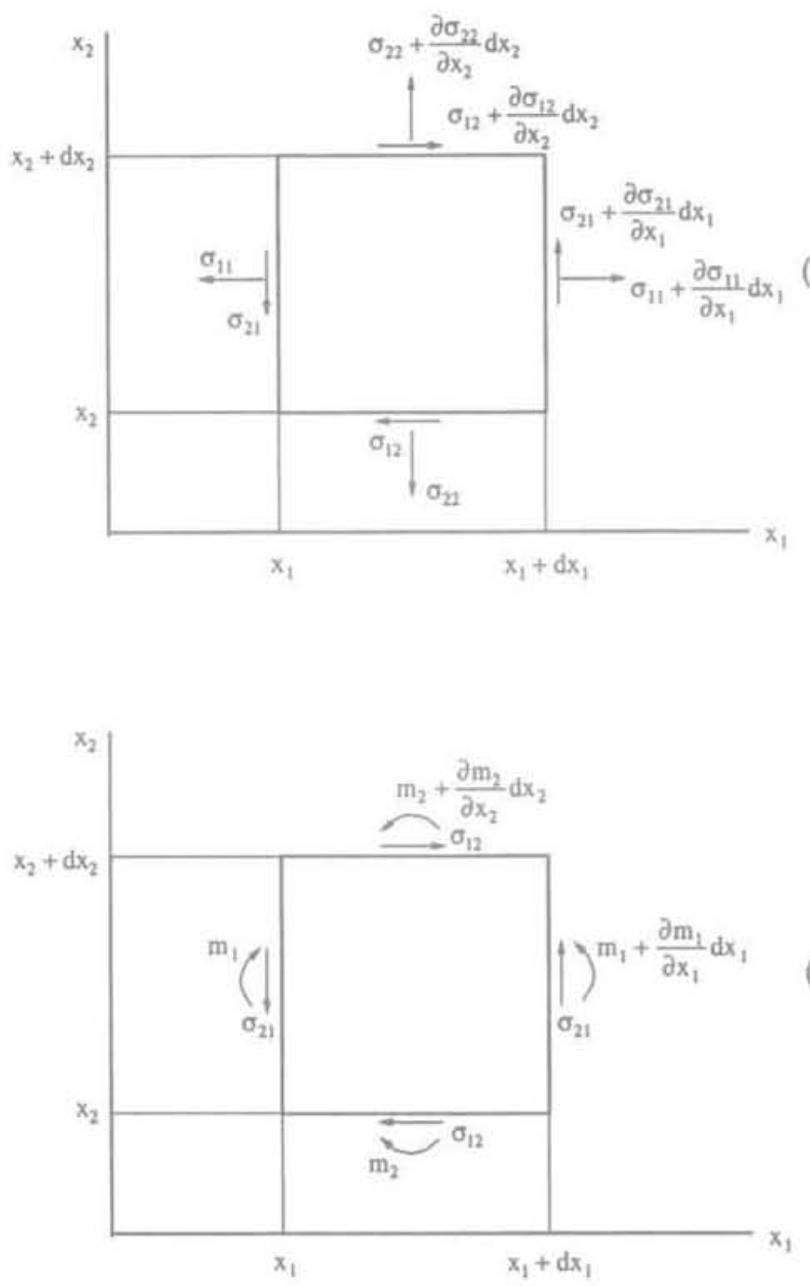

(b)

FG.4 Équation d'équilibre dans un milieu de Cosserat. (a) équilibre des forces ; (b) équilibre des moments.

rique. Un autre module de cisaillement $G$ (module de cisaillement de Cosserat) est introduit qui relie la partie antisymétrique de la déformation relative à la partie antisymétrique de la contrainte déviatorique. Enfin, les couples de contraintes sont reliés aux courbures correspondantes par un module de flexion $M$, qui a la dimension d'une force. Ainsi, dans un milieu de Cosserat bidimensionnel élastique isotrope deux paramètres supplémentaires sont introduits par rapport au milieu élastique classique (de Cauchy) : un module de cisaillement $G$ et une longueur interne $I=\sqrt{M / G}$. Les relations de comportement (15) peuvent être généralisées au cas d'un milieu élastique anisotrope :

$$
\begin{gathered}
\sigma_{11}=C_{11} \gamma_{11}+C_{12} \gamma_{22} \\
\sigma_{22}=C_{21} \gamma_{11}+C_{22} \gamma_{22} \\
\sigma_{12}=\left[G+G_{c}(1-\alpha)\right] \gamma_{12}+[G-G] \gamma_{21} \\
\sigma_{21}=\left[G-G_{2}\right] \gamma_{12}+\left[G+G_{c}(1+\alpha)\right] \gamma_{21} \\
m_{1}=M_{1} \kappa_{1} \\
m_{2}=M_{2} \kappa_{2}
\end{gathered}
$$

Dans les relations (16), $\alpha$ est un paramètre d'anisotropie. Les modules de flexion généralement différents $M_{1}$ et $M_{2}$ introduisent alors deux longueurs internes

$I_{1}=\sqrt{M_{1} / G}$ et $I_{2}=\sqrt{M_{2} / G}$. Un milieu de Cosserat possède donc une ou plusieurs longueurs caractéristiques 
(ou longueurs internes) dans la fomulation des relations de comportement. Ces longueurs internes sont reliées à la taille de la microstructure comme on le verra par la suite.

Les coefficients des relations de comportement (16) peuvent s'exprimer explicitement à partir des modules élastiques des couches individuelles supposées isotropes et des caractéristiques élastiques des joints. Pour ce faire, on considère différents chargements élémentaires du milieu stratifié (Zvolinskii et Shkhinek, 1984). Notons que les valeurs de ces constantes peuvent dépendre du choix de ces « expériences de référence ». Cette procédure conduit aux valeurs suivantes (cf. Mühlhaus, 1995; Adhikary et al., 1996) :

$$
\begin{aligned}
& C_{11}=\frac{E}{1-v^{2}-\frac{v^{2}(1+v)^{2}}{1-v^{2}+E /\left(k_{n}\right)}} ; C_{22}=\frac{(1-v) E}{(1+v)(1-2 v)+(1-v) E /\left(k_{n}\right)} \\
& C_{12}=C_{21}=\frac{v E}{(1+v)(1-2 v)+(1-v) E /\left(k_{n}\right)} \\
& G=\frac{1}{8} \frac{E}{(1+v)} \frac{5 k_{s}+E /(2(1+v))}{k_{s}+E /(2(1+v))} ; \quad G_{c}=\frac{E}{8(1+v)} ; \quad \alpha=2 \\
& M_{1}=\frac{E h^{2}}{12(1-2)}\left[\frac{E /(2(1+v))}{k_{s}+E /(2(1+v))}\right] ; M_{2}=0
\end{aligned}
$$

où E est le module d'Young et v le coefficient de Poisson de la roche intacte, $k_{n}$ et $k_{s}$ sont les raideurs normales et tangentielles des joints respectivement. Le couple de contraintes $\mathrm{m}_{2}$ est identiquement nul pour un matériau stratifié car la structure est unidirectionnelle. Lorsque les raideurs des joints $\left(k, k_{n}\right) \rightarrow \infty$, les discontinuités de déplacement disparaissent et l'on retrouve le milieu élastique, isotrope. On trouvera dans les travaux de Adhikary et al. (1999) une application de ce modèle de Cosserat pour l'étude d'une excavation dans un milieu stratifié.

\section{4}

\section{Un modèle de Cosserat pour les structures de blocs}

Pour des structures périodiques de blocs, qu'il s'agisse de murs de maçonnerie ou encore de blocs rocheux formés par un système régulier de joints dans un massif fracturé, la représentation par un milieu continu de Cosserat permet de prendre en compte les mouvements de rotation et de pivotement des blocs individuels, ce que ne permet pas l'homogénéisation par milieu continu classique.

On considère ici un modèle simple d'une structure de blocs représentée sur la figure 5, pour laquelle chaque bloc est entouré de six blocs adjacents. Par souci de simplicité, on suppose que l'élasticité des blocs et des joints est concentrée sur les arêtes des blocs.

\section{Joints élastiques}

Pour des joints élastiques on suppose que l'interaction entre les blocs est concentrée en six points (Fig. 5).
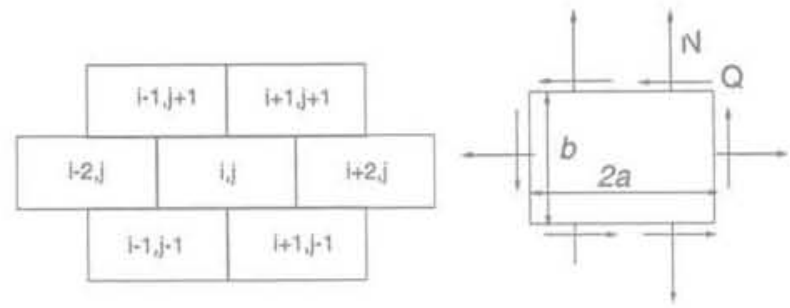

FiG.5 Un modèle de structure de blocs.

Les efforts tangentiels et normaux s'écrivent de la façon suivante en fonction du déplacement relatif des blocs

$$
\begin{aligned}
& Q_{k l}=c_{Q} \Delta u_{k l} \\
& N_{k l}=c_{N} \Delta v_{k l}
\end{aligned}
$$

où $c_{0}$ et $c_{N}$ sont les rigidités tangentielles et normales de l'interfäce respectivement, et les déplacements relatifs $\Delta u$ et $\Delta v$ aux différents points de contact sont donnés par les relations suivantes :

$$
\begin{aligned}
& \Delta u_{i \pm 1, j \pm 1}=u_{i \pm 1, j \pm 1}-u_{i, j} \pm \frac{b}{2}\left(\varphi_{i \pm 1, j \pm 1}+\varphi_{i, j}\right) \\
& \Delta u_{i \pm 2, j}=u_{i \pm 2, j}-u_{i, j} \\
& \Delta v_{i \pm 1, j \pm 1}=v_{i \pm 1, j \pm 1}-v_{i, j} \mp \frac{a}{2}\left(\varphi_{i \pm 1, j \pm 1}+\varphi_{i, j}\right) \\
& \Delta v_{i \pm 2, j}=v_{i \pm 2, j}-v_{i, j} \mp a\left(\varphi_{i \pm 2, j \neq 1}+\varphi_{i, j}\right)
\end{aligned}
$$

où $2 a$ et $b$ sont les dimensions du bloc, $u_{i j}, v_{i,}$ et $\varphi_{i,}$ sont les déplacements et la rotation du bloc numéroté $(i, j)$.

On obtient les paramètres élastiques du milieu continu de Cosserat équivalent (équation 16) en identifiant l'énergie élastique spécifique de la structure discrète et celle du milieu continu (cf. Sulem et Mühlhaus, 1997; Sab, 1996). Pour la structure discrète celle-ci s'écrit:

$$
E_{d}=\frac{1}{4 a b} \sum\left(Q_{k l} \Delta u_{k l}+N_{k l} \Delta v_{k l}\right)
$$

Pour le milieu continu de Cosserat elle s'écrit :

$$
E_{\mathrm{c}}=\sigma_{11} \gamma_{11}+\sigma_{12} \gamma_{12}+\sigma_{21} \gamma_{21}+\sigma_{22} \gamma_{2}+m_{1} \kappa_{1}+m_{2} k_{2}
$$

Par développement de Taylor de l'expression (23) et remplacement des quotients finis par des quotients différentiels, on peut identifier les deux expressions de l'énergie spécifique élastique, ce qui conduit à :

$$
\begin{aligned}
& C_{18}=\left(c_{Q}+2 c_{N}\right) \frac{a}{b} ; C_{22}=C_{N} \frac{b}{a} ; C_{12}=C_{21}=0 \\
& G=G_{c}=\frac{1}{4}\left[c_{Q} \frac{b}{a}+\left(c_{N}+2 c_{Q}\right) \frac{a}{b}\right] \\
& \alpha=2 \frac{a^{2}\left(c_{N}+2 c_{Q}\right)-c_{Q} b^{2}}{\alpha^{2}\left(c_{N}+2 c_{Q}\right)+c_{Q} b^{2}} \\
& M_{1}=\frac{a}{b}\left[C_{N} \frac{a^{2}}{4}+c_{Q}\left(\frac{b^{2}}{4}+2 a^{2}\right)\right]: M_{2}=\frac{b}{a}\left[c_{N} \frac{a^{2}}{4}+c_{Q} \frac{b^{2}}{4}\right]
\end{aligned}
$$

Le paramètre $C_{11}$ (resp. $C_{22}$ ) représente la rigidité du système pour une sollicitation uniaxiale dans la direction 1 (resp. 2). La nullité des paramètres $C_{i j}$ pour $i \neq j$ exprime qu'il n'y a pas d'“ effet de Poisson » pour ce modèle de bloc. Les paramètres de rigidité en flexion $M_{1}$ et $M_{2}$ font intervenir directement les dimensions des blocs : 
$\frac{M_{1}}{G}=a^{2} \frac{c_{N}+c_{Q}\left(8+(b / a)^{2}\right)}{c_{N}+c_{Q}\left(2+(b / a)^{2}\right)} ; \frac{M_{2}}{G}=b_{2} \frac{c_{N}+c_{Q}(b / a)^{2}}{c_{N}+c_{Q}\left(2+(b / a)^{2}\right.}$

On caractérise la réponse dynamique d'un milieu à partir de sa fonction de dispersion c'est-à-dire de la relation entre la longueur d'onde d'un signal et sa vitesse de propagation dans le milieu. Dans un milieu continu classique, étant donné qu'il n'y aucune longueur caractéristique dans la description du comportement, la fonction de dispersion est une constante, i.e. toutes les ondes se propagent à la même vitesse. Dans un milieu de Cosserat, l'existence d'une longueur interne permet de décrire une dépendance de la vitesse d'une onde en fonction de sa longueur d'onde. Sur la figure 6 on représente la fonction de dispersion des ondes de cisaillement du milieu discret et du milieu de Cosserat équivalent (la fonction de dispersion des ondes de compression dans un milieu de Cosserat est bien entendu une constante comme celle d'un milieu de Cauchy). Cette figure montre que le milieu équivalent de Cosserat donne une bonne approximation du milieu discret mème pour des longueurs d'onde de l'ordre de 4 à 5 fois la taille du bloc. Pour des grandes valeurs de la longueur d'onde, le signal " ne voit pas » la microstructure et le milieu continu classique donne une bonne approximation.

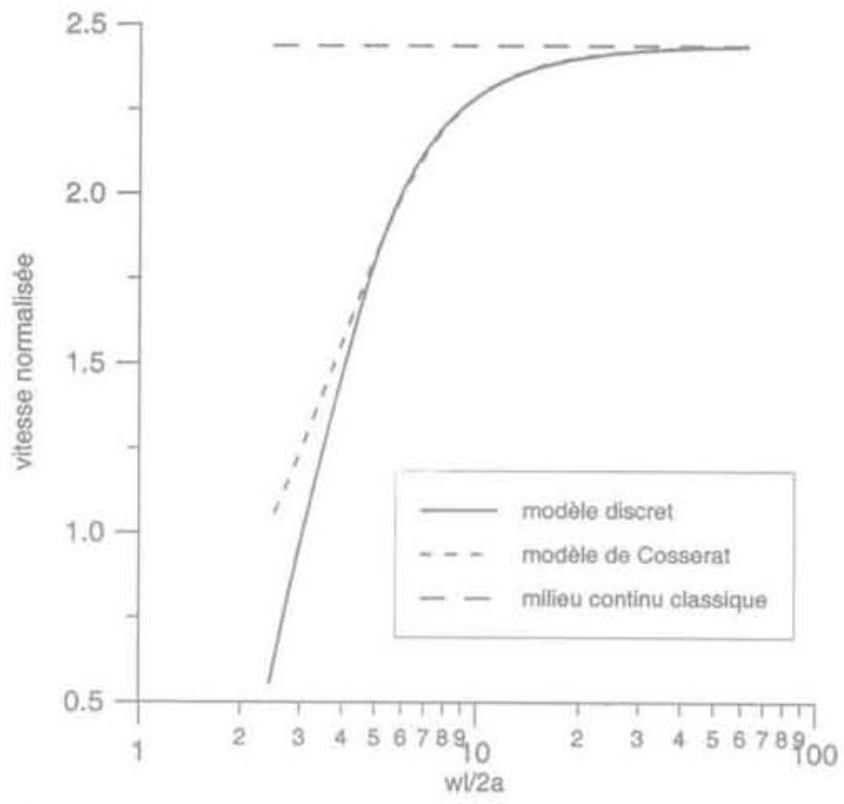

FIG, \& Comparaison de la fonction de dispersion des ondes de cisaillement. $\left(c_{\mathrm{s}}=10 \mathrm{c} ; \mathrm{b}=2 \mathrm{a}\right)$.

\section{Joints élastoplastiques}

On peut ètendre le milieu de Cosserat élastique défini ci-dessus à un milieu de Cosserat élastoplastique en considérant les différents mécanismes de plastification des joints (Mühlhaus, 1993; Sulem et Mühlhaus, 1997). Ces mécanismes sont essentiellement un mécanisme de glissement le long des joints et un mécanisme de pivotement des blocs. Par conséquent, il est nécessaire de considêrer simultanément plusieurs critères de plasticité. Pour le mécanisme de glissement, on considère simplement un critère de Coulomb. En notant négativement les contraintes de compression il s'écrit sous la forme :

$$
F^{1}=\left|\sigma_{12}\right|+\tan \phi \sigma_{22}-c \leq 0
$$

où c et $\phi$ sont respectivement la cohésion et l'angle de frottement des joints. Pour un mécanisme de frottement solide, l'hypothèse d'incompressibilité plastique est physiquement acceptable de sorte que le potentiel plastique s'exprime de la façon suivante:

$$
Q^{1}=\left|\sigma_{12}\right|
$$

Par ailleurs, un mécanisme de pivotement peut être décrit par un système de forces et de moments agissant sur un élément de volume sous la forme (Fig. 7):

$$
F^{(12.2)}=-N^{(1.2)}+\frac{2}{a}\left|M^{i 12 i}\right| \leq 0
$$

Pour le milieu de Cosserat correspondant, le critère de plasticité correspondant au mécanisme de pivotement s'écrit (Mühlhaus, 1993)

$$
F^{(2: 3)}=\sigma_{22} \pm \frac{b}{a} \sigma_{21}-\frac{2}{a}\left|m_{2} \pm \frac{b}{a} m_{1}\right| \leq 0
$$

avec une loi d'écoulement normale :

$$
Q^{(2,3)}=F^{(2,3)}=\sigma_{22} \pm \frac{b}{a} \sigma_{21}-\frac{2}{a}\left|m_{2} \pm \frac{b}{a} m_{1}\right| \leq 0
$$

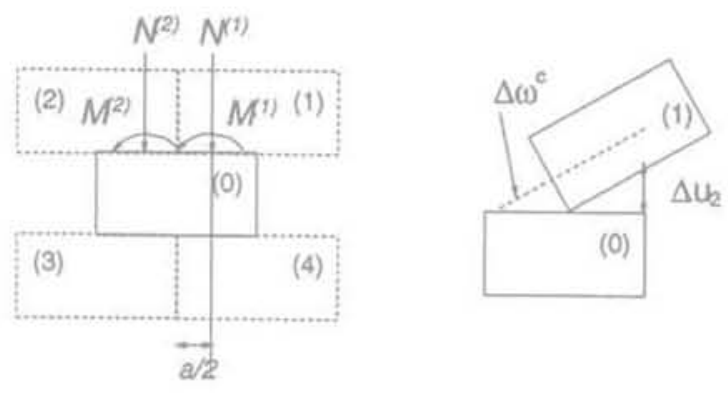

FGG.7 Conditions de pivotement.

\section{Formulation des équations constitutives incrémentales du modèle de Cosserat}

On définit les pseudo-vecteurs de contrainte et déformation généralisés par:

$$
\begin{aligned}
& s=\left\{\sigma_{11}, \sigma_{22}, \sigma_{12}, \sigma_{21}, m_{1}, m_{2}\right\} \\
& e=\left\langle\gamma_{11}, \gamma_{22}, \gamma_{12}, \gamma_{21}, \kappa_{1}, \kappa_{2}\right|
\end{aligned}
$$

La vitesse de déformation est décomposée de façon classique en une partie élastique et une partie plastique :

$$
\dot{e}=\dot{e}^{e}+\dot{e}^{p}
$$


Les relations d'élasticité (16) sont écrites sous la forme générale suivante :

$$
\dot{s}=C^{e} \dot{e}^{e}
$$

où $C^{e}$ est le tenseur de rigidité élastique.

La vitesse de déformation plastique est exprimée à partir de la loi d'écoulement:

$$
\dot{e}^{p}=\sum_{\alpha} \dot{\lambda}^{\alpha} \frac{\partial Q^{\alpha}}{\partial S}
$$

où les multiplicateurs plastiques $\lambda_{4}$ sont éliminés en utilisant les relations de consistance:

$$
F^{a}=0 \text { et } \dot{F}^{\alpha}=\left(\frac{\partial F^{\alpha}}{\partial s}\right)^{\top} \dot{s}+\left(\frac{\partial F^{\alpha}}{\partial e^{p}}\right)^{\top} \lambda^{\alpha} \frac{\partial Q^{\alpha}}{\partial s}=0
$$

Si un seul critère est activé et dans l'hypothèse d'un comportement plastique parfait on obtient les relations élastoplastiques incrémentales suivantes :

$$
\dot{s}_{i}=C_{m m} \dot{e}_{m}=C_{i k}^{e}\left[\delta_{k m} \frac{\frac{\partial \mathrm{Q}}{\partial \mathrm{s}_{\mathrm{k}}} C_{m}^{e} \frac{\partial F}{\partial \mathrm{s}_{\mathrm{n}}}}{\frac{\partial \mathrm{F}}{\partial \mathrm{s}_{\mathrm{r}}} C_{r}^{e} \frac{\partial \mathrm{Q}}{\partial \mathrm{s}_{s}}}\right] \dot{e} \text { ou } \dot{s}=\mathrm{Ce}
$$

5

\section{Mise en œuvre du modèle de Cosserat dans une formulation par éléments finis}

Ce modèle de Cosserat pour les structures de blocs a été introduit dans un programme d'éléments finis intitulé COSSBLPL pour la modélisation de la réponse statique et dynamique des structures de maçonnerie (Cerrolaza et al., 1999) en grandes déformations (voir aussi Adhikary et al,, 1999). On présente ici les aspects essentiels de la généralisation de la formulation par élémerits finis à un milieu de Cosserat.

On définit les pseudo-vecteurs de traction et déplacement généralisés :

$$
v=\left\{u_{4}, u_{2}, \omega_{3}^{c}\right\} ; f=\left\{t_{1}, t_{2}, m_{3}\right\}
$$

ce qui permet d'êcrire le principe des puissances virtuelles sous la même forme que le milieu continu classique :

$$
\int_{B} s^{T} \delta e d V=\int_{C B} f^{T} \delta v d A
$$

En introduisant les relations de comportement (37) on obtient :

$$
\int_{B} \Delta e^{T} C^{T} \delta e d V=\int_{i B} f^{T} \delta v d A-\int_{B} s_{f}^{T} \delta e d V
$$

La distribution des déplacements et de la rotation de Cosserat à l'intérieur du domaine $B^{e}$ d'un élément fini est exprimé à partir des fonctions d'interpolation $\Phi^{M}$

$$
v=\Phi^{M} v^{M} ; \delta v=\Phi^{M} \delta v^{M} \quad M=1,2 \ldots M^{\mathrm{F}}
$$

où $M^{c}$ est le nombre de points nodaux de l'élément fini considéré.
Le vecteur de déformation e s'exprime à partir des variables nodales:

$\mathrm{e}=B^{M} v^{M} ;\left[B^{M}\right]=\left[\begin{array}{lrr}\phi_{1}^{M} & 0 & 0 \\ 0 & \phi_{2}^{M} & 0 \\ \phi_{2}^{M} & 0 & \phi^{M} \\ 0 & \phi_{1}^{M} & -\phi^{M} \\ 0 & 0 & \phi_{1}^{M} \\ 0 & 0 & \phi_{2}^{M}\end{array}\right]$

En utilisant l'équation (42), l'équation (40) devient:

$$
\begin{aligned}
\left(\delta v^{M}\right)^{T} K^{M N} \Delta v^{N} & =\left(\delta v^{M}\right)^{T}\left(F_{\text {ext }}^{M}-F_{\text {int }}^{M}\right) \\
K^{M N} & =\int_{B^{e}}\left(B^{M}\right)^{T} C B^{N} d v
\end{aligned}
$$

est la matrice de rigidité tangente de l'élément :

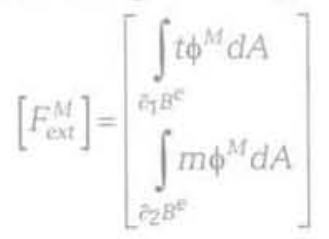

vecteur de forces appliquées généralisées et

$$
\left[F_{i n t}^{M}\right]=\int_{B^{e}}\left(B^{M}\right)^{T} s_{t} d V
$$

est le vecteur de contraintes généralisẻes.

\section{6}

\section{Application à l'étude de la réponse dynamique d'un mur du Parthénon}

Nous présentons ici comme application un exemple de calcul bidimensionnel par éléments finis de la réponse statique et dynamique d'une structure de maçonnerie. Il s'agit d'un mur du Parthénon étudié dans le cadre du projet « Environnement » EV5V-CT93-0300 de la Commission européenne (Schlosser et al., 1997).

\section{1}

\section{Géométrie et caractéristiques mécaniques de la structure}

On considère un mur d'une longueur de $50 \mathrm{~m}$ et d'une hauteur de $8,85 \mathrm{~m}$ constitué de blocs de marbre de Dionysos de $1,2 \mathrm{~m}$ de long, $0,52 \mathrm{~m}$ de haut et d'une épaisseur de $1,18 \mathrm{~m}$. Ce mur repose sur une base constituée de blocs de dimensions différentes (longueur $=2,36 \mathrm{~m}$, hauteur $=1,18 \mathrm{~m}$ ). Les caractéristiques mécaniques des joints ont été déterminées par le laboratoire 3S-IMG partenaire du projet (Armand et al., 1997) :

- rigidité normale : $k_{N}=14,35 \mathrm{~N} / \mathrm{mm}^{2}$;

- rigidité en cisaillement : $k_{Q}=0,94 \mathrm{~N} / \mathrm{mm}^{2}$;

- angle de frottement moyen : $35^{\circ}$. 
Le mur et la base sont représentés par un milieu continu équivalent constitué de deux matériaux de caractéristiques différentes :

- Matériau 1 (mur)

$C_{11}=19,165 \mathrm{MPa}$

$\mathrm{C}_{22}=6,97 \mathrm{MPa}$

$C_{12}^{22}=0$

$\mathrm{C}_{21}=0$

$G=2,74 \mathrm{MPa}$

$G_{c}=2,74 \mathrm{MPa}$

$\alpha=1,83$

$M_{1}=1,317 \mathrm{MPa} \cdot \mathrm{m}^{2}$

$M_{2}=0,658 \mathrm{MPa} \cdot \mathrm{m}^{2}$

- Matériau 2 (base)

$C_{n}=35,114 \mathrm{MPa}$

$C_{23}=17 \mathrm{MPa}$

$C_{12}=0$

$\mathrm{C}_{21}^{21}=0$

$G=5,09 \mathrm{MPa}$

$G_{c}=5,09 \mathrm{MPa}$

$\alpha=1,78$

$M_{3}=9,487 \mathrm{MPa}^{2}$

$M_{2}=6,359 \mathrm{MPa} \cdot \mathrm{m}^{2}$

\section{2}

\section{Modèle d'éléments finis}

Le modèle d'éléments finis comporte 245 éléments quadrangulaires à 4 nceudis et 285 nœuds au total (Fig. 8a). La topologie des éléments finis est indépendante de la géométrie des blocs. Les zones corres- pondant aux deux matériaux différents sont repérées par les nombres (1) pour le mur et (2) pour la base. Les conditions aux limites sont représentées sur la figure $8 \mathrm{~b}$ et expriment un appui rigide du mur sur le sol et une interaction élastique du mur avec la superstructure.

\section{3}

\section{Réponse statique et dynamique}

Le chargement comporte le poids propre du mur (densité du marbre : $2700 \mathrm{~kg} / \mathrm{m}^{3}$ ) et un chargement à la base de $2400 \mathrm{kN}$ uniformément distribué à la base (Fig. 8c). La déformée élastique de la structure est représentée sur la figure 9 .

Le champ de déplacement est uniforme dans la direction horizontale. Il est maximum à la base où le chargement est appliqué.

La réponse dynamique élastique est étudiée par l'application du chargement précédent cyclé sinusoïdalement avec une gamme de fréquences allant de 0,2 à $10 \mathrm{~Hz}$. Les inerties en rotation des deux matériaux sont: - matériau $1: J_{4}=384,84 \mathrm{~kg} / \mathrm{m}$; - matériau $2: J_{2}=1579,75 \mathrm{~kg} / \mathrm{m}$.

Les résultats de calculs sont illustrés à partir de la réponse de quelques nœuds caractéristiques représentés sur la figure $10 \mathrm{a}$. Ces résultats sont résumés sur la figure $10 \mathrm{~b}$ où le facteur d'amplification défini comme le rapport entre l'amplitude maximale du déplacement horizontal sous chargement sinusoïdal durant dix cycles est représenté en fonction de la fréquence du signal. Ce graphe montre que le facteur d'amplification décroit avec la fréquence du signal.
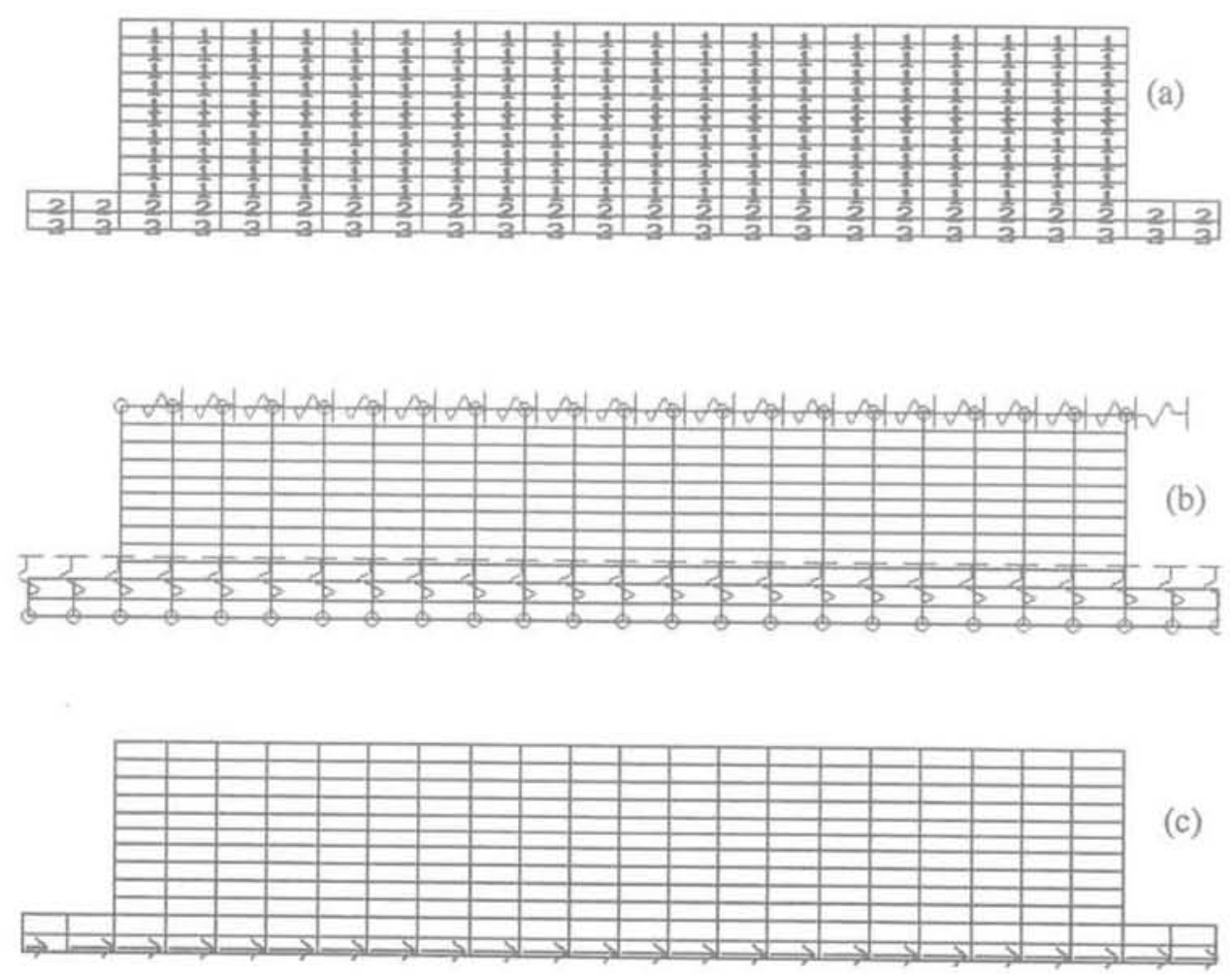

FIG.8 Modèle par éléments finis d'un mur du Parthénon : (a) maillage, (b) conditions aux limites, (c) chargement. 


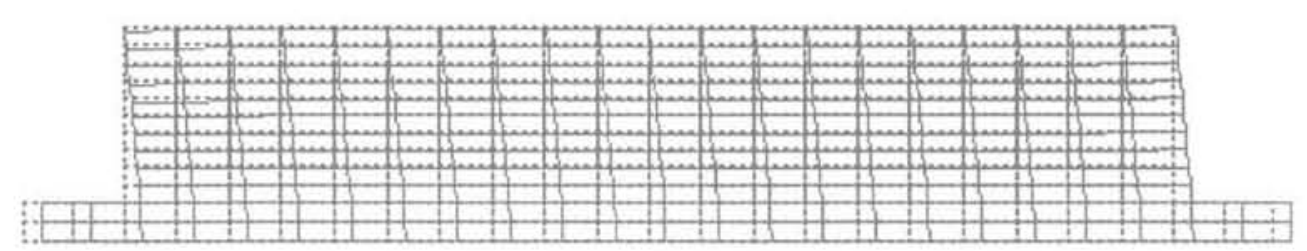

FG.9 Déformée élastique.
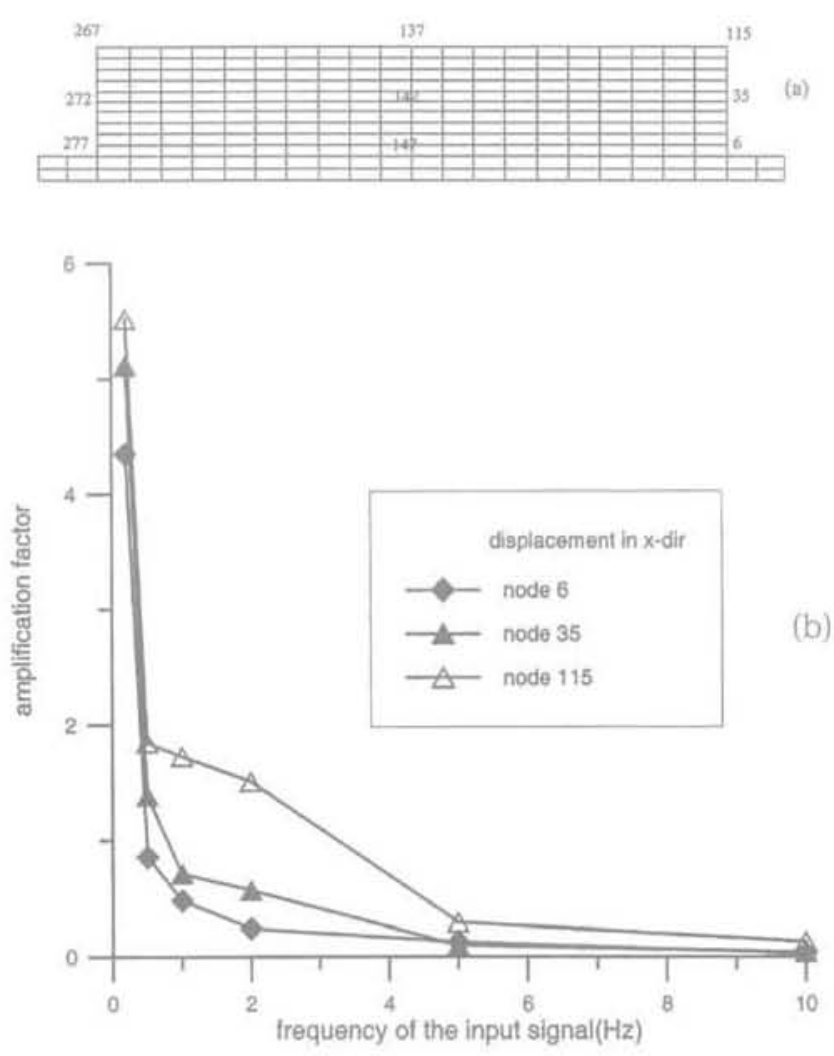

FG. 10 (a) position des nœuds caractéristiques ; (b) facteur d'amplification de la réponse horizontale.

\section{Exemples d'application en géotechnique}

Pour l'étude de problèmes de géotechnique en milieu rocheux fracturé périodique, T'approche par milieu continu de Cosserat permet de distinguer les différents modes de rupture, par glissement et/ou par pivotement. On trouvera une application de la théorie de Cosserat aux tunnels excavés dans des massifs fracturés dans l'article de Dai et al. (1996). L'objet des deux exemples bidimensionnels que nous présentons ici est de mettre en évidence l'existence de différents modes possibles de plastification pour l'étude d'une fondation souple et pour celle de la stabilité d'une pente.

\section{1}

\section{Fondation souple sur un massif de blocs}

On considère un espace semi-infini soumis à une sollicitation normale uniforme sur une longueur L. Ceci peut représenter l'exemple classique d'une semelle superficielle souple sur un massif de blocs rocheux (Fig. 11).

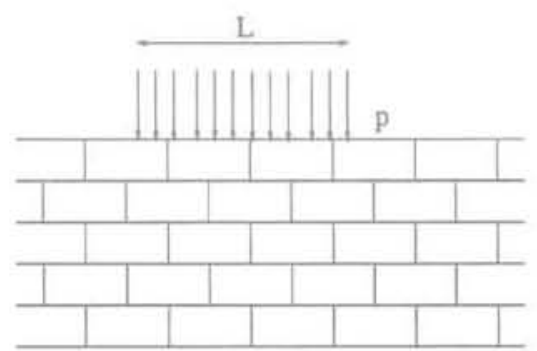

FIG.11 Fondation superficielle souple sur une structure périodique de blocs.

Pour les applications numériques, on considère l'ensemble des paramètres géométriques et mécaniques suivant :

- caractéristiques des blocs :

- longueur : $2 a=1 \mathrm{~m}$,

- largeur : $b=0,5 \mathrm{~m}$;

- caractéristiques des joints :

- rigidité : $C_{N}=c_{Q}=1 \mathrm{GPa}$;

- angle de frottement : $\Phi=20^{\circ}$;

- cohésion : $c=1 \mathrm{kPa}$;

- angle de dilatance : $\psi=0^{\circ}$;

- longueur de la semelle : $\mathrm{L}=10 \mathrm{~m}$.

Pour des raisons de symétrie, seule la moitié de la structure est maillée par éléments finis. Sur la figure 12a (resp. 12b) les zones plastiques pour le critère de glissement (resp. critère de pivotement) sont représentées en couleur sombre. Ces résultats montrent clairement que le critère de pivotement est atteint à la surface au voisinage des extrémités de la semelle de fondation sur une étendue limitée alors que le critère de glissement se développe largement en profondeur à l'intérieur du massif avec une orientation d'environ $30^{\circ}$ par rapport à l'horizontale. Par comparaison nous représentons sur la figure 13 les zones plastiques obtenues dans le cas d'un massif élastoplastique isotrope classique avec un critère de Mohr-Coulomb pour lequel les paramètres géomécaniques sont ceux d'une 

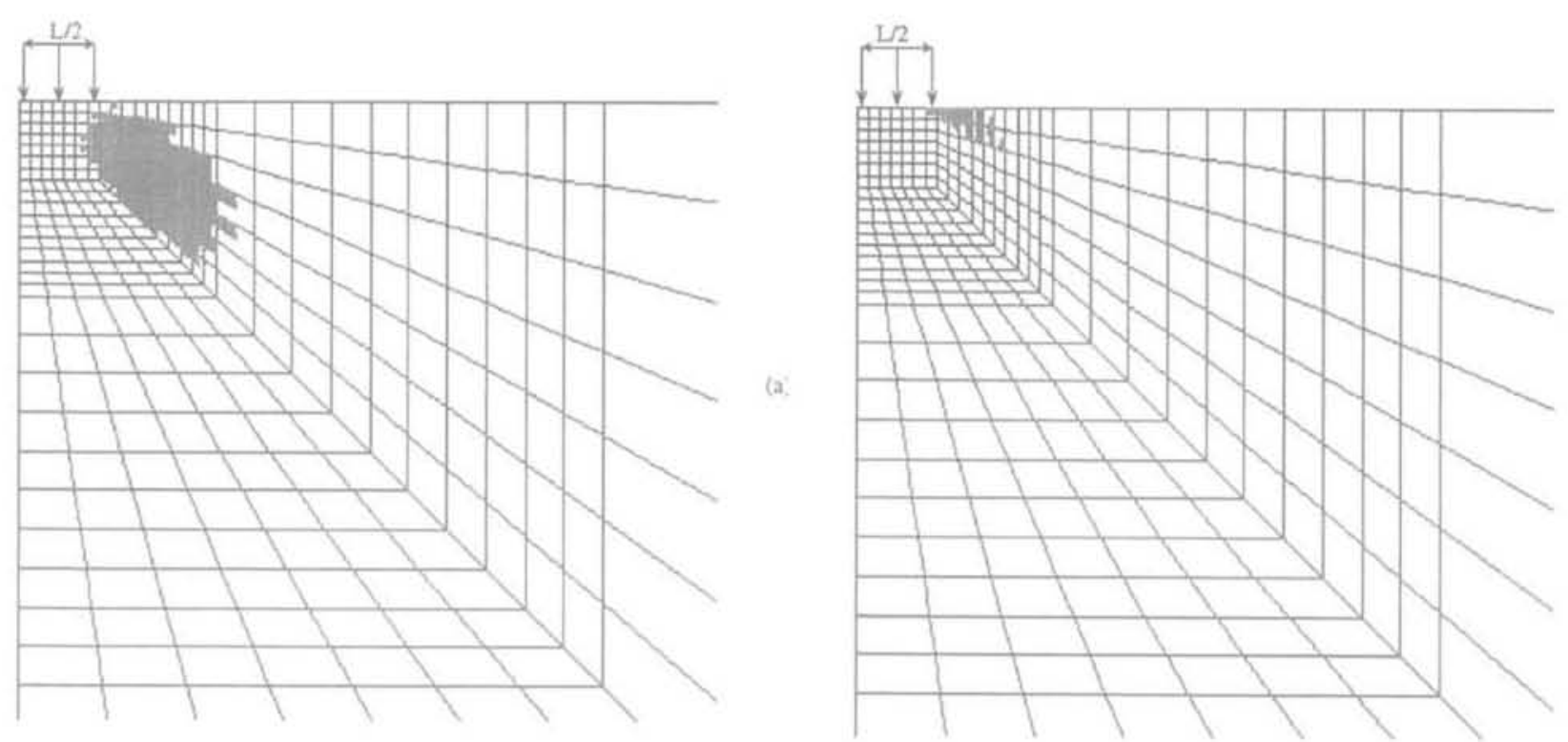

(b)

Fi6.12 Chargement normal uniforme sur une structure de blocs ; (a) zones de glissement (pression de 8,4 kPa), (b) zones de pivotement (pression de $4,5 \mathrm{kPa}$ ).

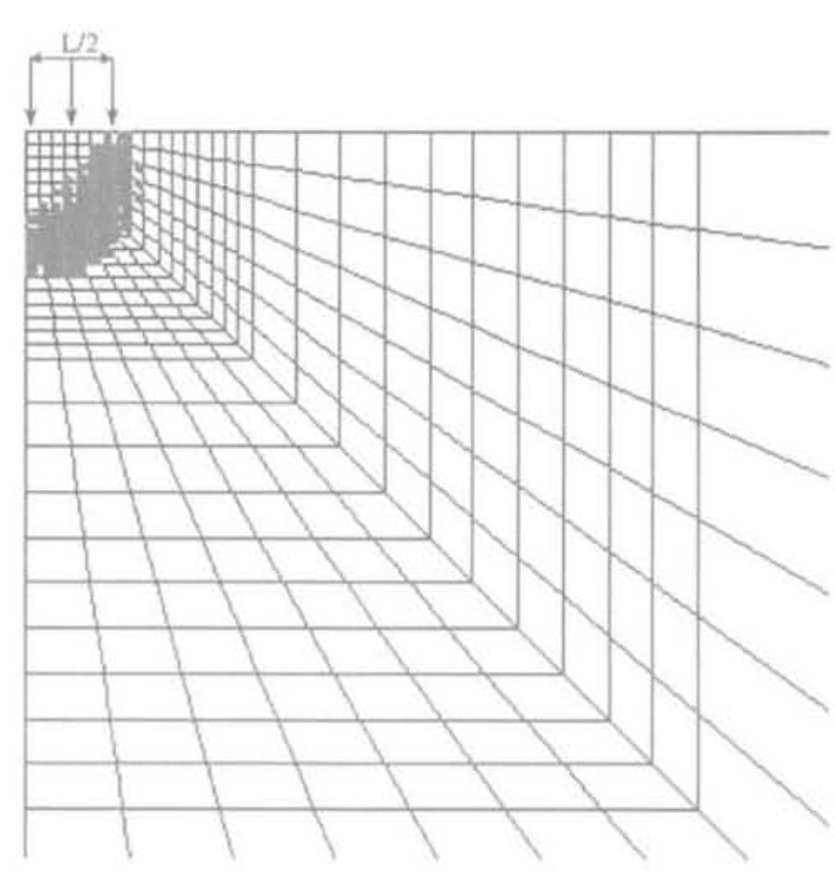

FG. 13 Zones plastiques pour un chargement normal uniforme avec un critère isotrope de Mohr-Coulomb.

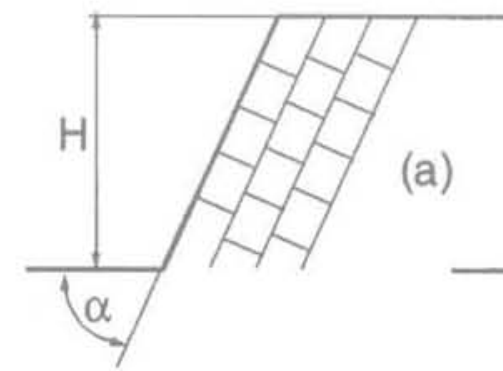

grave (module d'Young $=25 \mathrm{MPa}$, coefficient de Poisson $=0,3$, angle de frottement $=40^{\circ}$, cohésion nulle), Dans ce dernier cas, on retrouve la formation classique du cône élastique sous la fondation et le développement de la zone plastique sous celui-ci.

\section{2}

\section{Stabilité des pentes dans un massif rocheux}

On considère la configuration présentée sur la figure 14 pour laquelle deux cas extrêmes sont étudiés: (a) l'orientation des blocs est parallèle à la pente, (b) lorientation des blocs est parallèle à la surface. Pour les applications numériques on considère les mêmes caractéristiques géométriques et mécaniques des blocs que dans l'exemple précédent (\$ 7.1) et une pente de hauteur $\mathrm{H}=15 \mathrm{~m}$ et d'une inclinaison $\alpha=50^{\circ}$ par rapport à 1 horizontale.

Un avantage important de l'approche continue est qu'un même maillage par éléments finis nous permet d'étudier les deux configurations géométriques différentes; seuls les paramètres mécaniques du milieu de Cosserat équivalent diffèrent. Sur la figure 15 nous présentons le maillage de la structure constitué de quadrilatères à 4 noeuds. Sur les frontières $\mathrm{AB}$ et DC, on impose une condition de déplacement horizontal nul et sur la frontière $\mathrm{BC}$ une condition de déplacement vertical nul.

FiG. 14 Configurations géométriques d'une pente rocheuse : (a) blocs parallèles à la pente, (b) blocs parallèles à la surface. 


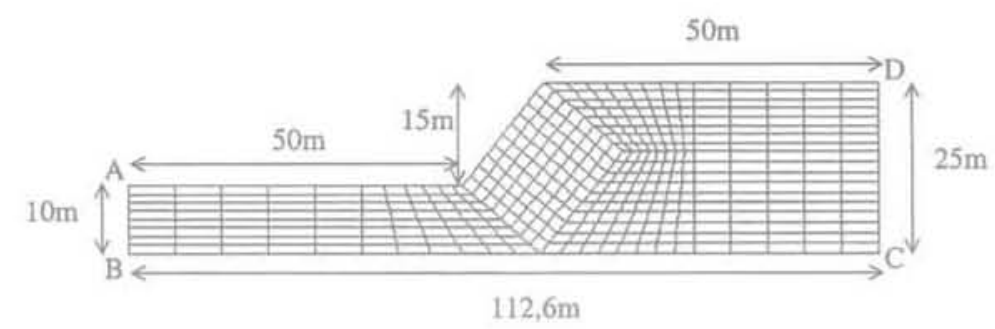

FG. 1s Modèle par éléments finis d'une pente.

\section{Configuration (a)}

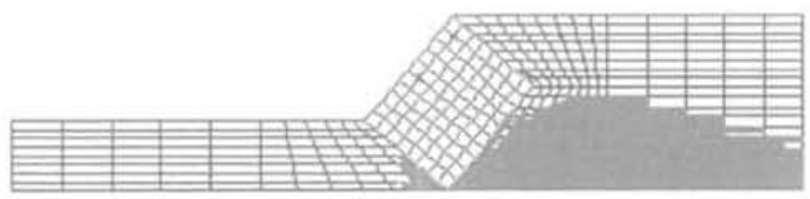

Zones de glissement

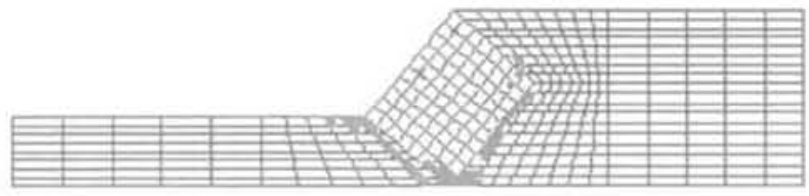

Zones de pivotement

Configuration (b)

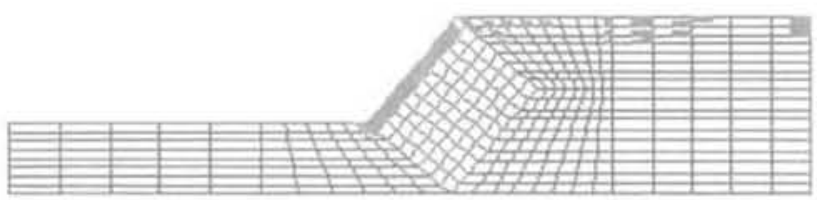

\section{Zones de glissement}

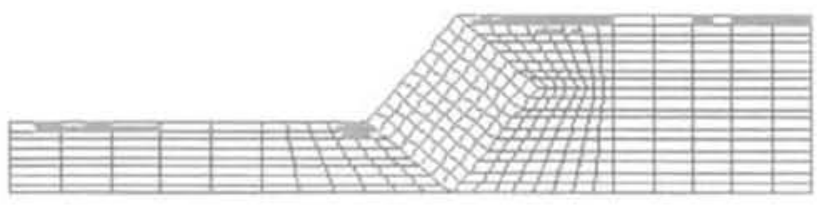

Zones de pivotement

FiG. 16 Zones plastiques

pour chaque configuration.
Les différents critères de plasticité par glissement ou pivotement sont étudiés successivement pour le massif rocheux sous poids propre dans l'hypothèse d'un écrouissage nul. Les résultats sont résumés sur la figure 16, où les zones de rupture sont représentées de couleur sombre. De façon évidente, la configuration (a) est plus critique que la configuration (b) pour le critère de glissement. Par ailleurs pour la configuration (b) une rupture par glissement apparaît le long de la pente alors qu'une rupture en pivotement apparait en surface.

\section{8}

\section{Conclusion}

La représentation d'un massif rocheux montrant un réseau régulier de joints par un milieu continu équivalent de Cosserat est présentée ici comme une alternative aux approches de type discret. L'homogénéisation est présentée pour les structures stratifiées et les structures de blocs. La cinématique enrichie du milieu de Cosserat permet de prendire en compte la rotation individuelle des blocs ou la flexion individuelle de chaque couche et d'introduire directement dans la loi de comportement des caractéristiques géométriques de la structure élémentaire telles que la taille et l'orientation des blocs ou l'épaisseur des couches. A partir de l'évaluation de la fonction de dispersion d'une structure de blocs, on a montré que cette approche permet d'étendre le domaine de validité de l'homogénéisation à des structures comportant un faible nombre de blocs. Ainsi les fonctions de dispersion du milieu discret et du milieu continu de Cosserat équivalent sont sensiblement égales pour une longueur d'onde du signal supérieure à 5 fois la taille des blocs.

Différents critères de plasticité correspondant à des mécanismes différents tels que le glissement entre blocs et le pivotement des blocs sont formulés et mis en œuvre dans une approche par éléments finis. Un même maillage peut être alors utilisé pour différentes configurations de taille ou d'orientation des blocs. Les exemples d'une structure de maçonnerie sous sollicitation dynamique, d'une fondation souple et d'une pente rocheuse sous sollicitation statique illustrent cette approche. Les différentes zones de rupture correspondant aux différents critères sont déterminées. 
Cette représentation d'un milieu discontinu par un milieu continu équivalent de Cosserat présente des avantages clairs de simplicité de mise en œuvre des calculs numériques par rapport à l'approche explicite par modèle discret. Elle est cependant limitée au cas d'une distribution fixe et régulière de joints orthogonaux et c'est sa principale limite. Ce type de configuration est cependant couramment observé dans les massifs rocheux. La formation de nouvelles fractures en cours de chargement peut être prise en compte par l'introduction de surfaces de discontinuités dans le modèle.
Cette approche est, pour l'instant, limitée au cas bidimensionnel. Elle sera prochainement étendue au cas tridimensionnel afin de prendre en compte les différents modes de rotation des blocs suivant différents axes.

\section{REMERCIEMENTS}

Une partie de ce travail a été possible arâce au financement de la Commission européenne dans le cadre du proiet Environnement $N^{\circ}$ EV5V-CT93-0300 que les auteurs souhaitent remercier ici, ainsi que tous les partenaires clu projet.

\section{$\overline{\text { Bibliographie }}$}

Adhikary D.P. Dyskin A.V. Jewell, R.J. * Numerical modelling of the flexural deformation of foliated rock slopes $m$. Int. I. Rock Mech. Min. Sci. and Geomech. Abstr. 33,6, 1996, p. 595-606

Adhikary D.P. Mühlhaus H.B. Dyskin AV - - Modelling the large deformations in stratified media. The Cosserat continuum approach 1 . Mechanics of Cohesive-Frictional Mat. 4, 1999, p. 195-213.

Armand G., Boulon M., Jay J.-C., Papadopoulos C. Picaud G., Reveillet M.. Rev B., Verdys, J.F. - Final report for CEC, Environment project a Monuments under seismic action y, Contrat EV5VCT93-0300. 1997.

Bakhvalov N. Panasenko G. - Homogenisation : averaging processes in periodic media, Dordrecht. The Netherlandis, Kluwer Academic publ. 1989.

Cerrolaza M., Sulem J., El Bied A. - u A Cosserat non-linear finite element ana. lysis software for blocky structures ग. Int. J. of Advances in Eng. Soft, Elsevier, 30. 1999 , P. $69-83$

Cundall P.A., Hart R. - « Numerical modelling of discontinua 1). Engng. Computa. tions, 9, 1992, p. 101-113.
Cosserat $\mathrm{E}_{\text {. Cosserat }} \mathrm{F}$ - Théorie des corps déformables. Paris, Hermann et fils. 1909.

Germain P. - u La méthode des puissances virtuelles en mécanique des milieux continus m. Part. I Journal de Mécanique. 12. 1973a, p. 235-274.

Germain P. - a The method of virtual power in continuum mechanics »). Part2. Microstructure. SIAM, J. Appl. Math., 25, 1973b, p. 556-575.

Mindlin R.D. - « Microstructure in linear elasticity 1, Arch. Rat. Mech. Anal. 4, 1964, p. 50-78.

Mühlhaus H.B. - " Continuum models for layered for layered and blocky rock n. In: Comprehensive Rock Engng., vol. 2 (Charles Fairhurst ed.) Pergamon Press, 1993. p. 209-230.

Mûhlhaus H.B. - " A relative gradient model for laminated materials $\mathrm{p}$. In : Continuum Models for Materials with Micro-Structure (Mühlhaus H.B. ed.), J. Wiley, chap. 13, 1995, p. 450-482.

Sab K. - « Déformations microscopiqués et macroscopiques dans un assemblage dense de particules rigides v. C. R. Acao Sci. Paris, t. 322, Série lib, 1996, p. 715-721.
Salamon MDG - uf Flastic moduli of stra tified rock mass v. Int. J. Rock Mech. Min. Sci. and Geomech. Abstr.. 5, 1968, p. 519-527.

Schaefer $H_{.}-\alpha$ Versuch einer Elastizitătstheorie des zweidimensionalen ebenen Cosserat-Kontinuums in, In: Miszellannenn der Angewandien Mekanik. Berlin. Akademie Veriag, 1962, p. 277-292.

Schlosser F. Sulem J. Frank R. El Bied A. - « Analytical and numerical modelling of structural response. Continuous models for discrete blocky structures i. Final report for CEC, Environment project \& Monuments under seismic action Contrat EV5V-CT93-0300, 1997.

Sulem J Mühlhaus H B - " A continuum model for periodic two-dimensional block structures 1 . Mechanics of cohesive-frictiona! materials, 2, 1997, p. 3146

Vardoulakis 1., Sulem J. - Bifurcation Analysis in Geomechanics, Glasgow. Blackie Academic \& Professional, 1995.

Zvolinskii N.V., Shkhinek, K.N. - "Continual model of laminar elastic medium y. Mechanics of Solids, 19, 1984, p. 1-9. 